\title{
Povidone-Iodine as Subgingival Irrigation in Chronic Periodontitis Treatment
}

\author{
Anh Thi Mai Nguyen ${ }^{1}$, Nhat Dinh Phan ${ }^{1}$, Thuy Anh Vu Pham²* \\ ${ }^{1}$ Faculty of Odonto-Stomatology, University of Medicine and Pharmacy Ho Chi Minh City, 652 \\ Nguyen Trai Str, District 5, Ho Chi Minh City, Vietnam \\ ${ }^{2}$ Department of Periodontology, Faculty of Odonto-Stomatology, University of Medicine and \\ Pharmacy Ho Chi Minh City, 652 Nguyen Trai Str, District 5, Ho Chi Minh City, Vietnam \\ *Email: pavthuy@ump.edu.vn
}

\begin{abstract}
The objective of this study is to verify the effects of $0.1 \%$ povidone-iodine (PVP-I) as subgingival irrigation on chronic periodontitis patients after 4, 6 and 8-week treatment. A clinical trial was conducted on 18 patients from 30 to 65 years old (mean age: $51.1 \pm 11.2$ ) with moderate or severe chronic periodontitis at Faculty of Odonto-Stomatology at University of Medicine and Pharmacy, Ho Chi Minh City, Vietnam 2017. Treatments of chronic periodontitis included oral hygiene instruction, scaling and root planing plus $0.1 \%$ PVP-I irrigation. Clinical parameters including plaque index (PI), gingival index (GI), bleeding on probing (BOP), pocket depth (PD), clinical attachment loss (CAL) and the bacteria level in subgingival plaque (BANA test) were evaluated at 4-time points: T0 (the initial visit), T4 (4 weeks after the initial visit), T6 (6 weeks after the initial visit) and T8 ( 8 weeks after the initial visit). The whole mouth (PI, GI, BOP, PD and CAL) parameters and BANA test score showed statistically significant reductions from $\mathrm{T} 0$ to T8 ( $\mathrm{p}<0.001)$. The PD and CAL of moderately deep ( $\mathrm{PD} \geqslant 5 \mathrm{~mm}$ and $<7 \mathrm{~mm}$ ); and deep pockets $(\mathrm{PD} \geqslant 7 \mathrm{~mm})$ were significantly decreased from $\mathrm{T} 0$ to $\mathrm{T} 8$ $(\mathrm{p}<0.001)$. The findings from the present study demonstrated that the topical application of $0.1 \%$ PVP-I, used as subgingival irrigation along with subgingival root debridement had beneficial influences on chronic periodontitis treatments.
\end{abstract}

Keywords: chronic periodontitis, povidone-iodine, subgingival irrigation

\section{Introduction}

Periodontitis is a chronic inflammatory disease which results in the destruction of connective tissues and structures surrounding the teeth. Its particular characteristic demonstrates in periodontal pocket formation and/or clinical attachment loss which is caused by specific bacteria existing in dental plaque [1]. If it is left untreated, the disease can cause loss of tooth supporting tissues, alveolar bones and periodontal ligaments and eventually, results in edentulism, posing a great negative impact on individuals' quality of life. Hence the global epidemiological data suggests periodontal disease to be one of a major burden on oral diseases [2].

The mouth, like all external surfaces of the body and the gut, has a substantial microflora living in symbiosis with a healthy host. The microflora of the mouth contains hundreds of species of aerobic and anaerobic bacteria. These organisms grow on tooth surfaces as complex, mixed, interdependent colonies in biofilms, and 
are attached and densely packed against the tooth in the deeper layers, with more motile forms in the superficial layers [3]. As dental plaque matures to a state that is associated with periodontal disease, the number of gram-negative and anaerobic bacteria increases [4-7]. Socransky and Haffajee (2005) [8] have catalogued and stratified the microbiota into groups or complexes, representing bacterial consortia that appear to occur together and that are associated with the biofilms of gingival health, gingivitis and periodontitis. The different microbial complexes have been associated with the sequence of colonization on the tooth surface as well as with disease severity. The "red complex", which appears later in biofilm development, comprises species that are considered periodontal pathogens, namely, Porphyromonas gingivalis, Treponema denticola, and Tannerella forsythia. These investigators have suggested that the "red complex" presents as a portion of the climax community in the biofilms at sites expressing periodontitis [9].

The main goal of periodontitis treatments is to eliminate disease-induced bacteria in dental plaque on the tooth surface. However, there still are many obstacles due to its complicated pathogenesis. An enormous research effort has been devoted to the study of periodontal-disease-associated microflora, from classic cultural methods to modern approaches on the molecular, whole genomic, and proteomic level $[10,11]$. The beneficial effects of scaling and root planing, combined with personal plaque control, in the treatment of periodontitis have been well documented. It can be considered as the golden criteria in periodontitis treatment. These procedures can result in reduction of clinical inflammation, microbial shifts to a less pathogenic subgingival flora, probing depth (PD) reduction, and gain of clinical attachment [12-15]. The goal of periodontal debridement is to produce a root that is biologically acceptable for a healthy attachment. However, as a multifactorial infection, periodontitis treatment might require more than these mechanical treating methods. They might be ineffective due to the limited access and visibility of the hand instruments in subgingival areas [16] or tissue-attaching characteristic of some particular bacteria species [17]. Therefore, the value of using antimicrobial agents such as povidone-iodine (PVP-I) to augment mechanical periodontal debridement should also be well considered [18]. Povidone-iodine is a cheap broad spectrum antiseptic agent frequently used in the therapy of periodontitis [19], especially in Scandinavia (Rosling et al. 2001) [20] and Switzerland (Sahrmann et al. 2014) [21]. Its spectrum of action covers bacteria associated with periodontitis (Reimer et al. 1998) [22] and its use as a rinse during initial periodontal therapy has been proven to provide a significant therapeutic benefit in terms of pocket depth reduction (Sahrmann et al. 2014) [21].

Considering the broad spectrum of antimicrobial activity, low financial cost, and good clinical results with PVP-I used in periodontal treatment, as well as the successes achieved with the association of periodontal debridement and antimicrobial agents 18, the aim of the present study was to verify the effects of 0.1 $\%$ PVP-I as a subgingival irrigation on chronic periodontitis patients after 4, 6 and 8-week treatment. 


\section{Materials and Methods}

\subsection{Subjects}

Eighteen patients from 30 to 65 years old (mean age: $51.1 \pm 11.2$ ) involved in this study were referred to the Faculty of Odonto-Stomatology, University of Medicine and Pharmacy of Ho Chi Minh City, Vietnam. Inclusive criteria: 1) Patients had at least 3 teeth which were diagnosed with moderate or severe chronic periodontitis according to standards of The American Academy of Periodontology (AAP) (2015): having at least 1 gingival bleeding site, periodontal pocket depth $\geqslant 5 \mathrm{~mm}$ or clinical attachment loss $\geqslant 3 \mathrm{~mm}$ and alveolar bone resorption in radiographic $\geqslant 16 \%$ or $>3 \mathrm{~mm}$ of tooth root. 2) Patients who have at least 8 sites which have periodontal pocket depth $\geqslant 5 \mathrm{~mm}$. Excusive criteria: 1) Patients who left during research process or didn't follow instructions during research process. 2) Patients with aggressive periodontitis or periodontitis associated with endodontics lesions. 3) Patients who recently have periodontal treatment within 12 months. 4) Patients who have acute oral inflammation. 5) Patients who have allergic reaction with iodine (changing sense of smell and taste, burn, vomiting when gargling with PVP-I). 6) Patients who recently use antibiotics and anti-inflammation drugs within 3 months. 7) Patients who have general disorders and risk factors (such as diabetes, cardiovascular diseases, HIV, smoking...). 8) Pregnant women or patients who use hormonal products. The study protocol was approved by the University of Medicine and Pharmacy, Ho Chi Minh City, Vietnam

\subsection{Treatment of periodontitis, periodontal examination and BANA test}

Treatments of chronic periodontitis including oral hygiene instruction, scaling and root planing plus $0.1 \%$ PVP-I irrigation; and periodontal examination of all subjects were performed by one dentist. Periodontal parameters including plaque index (PI, Silness \& Löe - 1967), gingival index (GI, Silness \& Löe - 1963), bleeding on probing (BOP), pocket depth (PD), clinical attachment loss (CAL) and the bacteria level in subgingival plaque (BANA test) were evaluated at 4-time points: T0 (the initial visit), T4 (4 weeks after the initial visit), T6 (6 weeks after the initial visit) and T8 ( 8 weeks after the initial visit). Pocket depth and clinical attachment loss were evaluated at six sites on each tooth using a Williams $1 \mathrm{~mm}$ scaled periodontal probe. Gingival bleeding on probing was assessed as presence or absence in $30 \mathrm{~s}$ after probing. All the teeth excluding the third molars were examined.

The presence of putative pathogens, P. gingivalis, T. denticola and T. forsythia in subgingival plaque was detected based on their ability to hydrolyze the synthetic trypsin substrate, named N-benzoyl-DL-arginine- 2-napthilamide (BANA) [23] by the BANA test (BANAMet LLC, Ann Arbor, MI, USA). Three samples of subgingival plaque taken from 3 deepest sites of 3 different teeth were immediately placed on the lower portion of the BANA test strip, while the upper portion was moistened with distilled water. The strip was folded over at the crease mark so that they contacted each other, and placed in an incubator for $5 \mathrm{~min}$ at $35^{\circ} \mathrm{C}$. The BANA test scores were recorded as follows: 0 (negative) when no blue color was visible; 1 (weak positive) when a faint blue color was detected; and 2 (positive) when a distinct blue color appeared. 


\subsection{Statistical Analysis}

The impact of the treatment strategy on the changes in PI, BOP, GI, PD, CAL and BANA test score (for baseline versus follow-up visit) was examined by repeat analysis of variance (ANOVA) combined with Greenhouse-Geisser correction and Bonferroni correction. The statistical analysis was performed with the SPSS software (version 22, IBM, NY). For all the analyses, a significance level of 5\% was used.

\section{Results}

\subsection{Whole mouth PI, GI and BOP}

At T0, the mean PI, GI and BOP of the group were recorded as $1.11 \pm 0.32$; $1.42 \pm 0.33$ and $46.15 \pm 24.8$. At T8, all patients showed a significant improvement in oral hygiene. Thus, the PI was reduced from $1.11 \pm 0.32$ at T0 to $0.66 \pm 0.21$ at $\mathrm{T} 8$ (Table 1). Furthermore, there was also a remarkable decrease in gingival inflammation. Both changes in GI and BOP from baseline to T4, T6 and T8 were statistically significant. In the details, the GI has changed from $1.42 \pm 0.33$ at T0 to $0,95 \pm 0,34$ at T8 and BOP has changed from $46.15 \pm 24.8$ at T0 to $23.30 \pm 10.6$ at T8 (Table 1).

Table 1. Whole mouth periodontal parameters (PI, GI, BOP) (mean \pm SD)

\begin{tabular}{|c|c|c|c|c|c|c|c|}
\hline & T0 & T4 & $\mathbf{p}$ & T6 & p & T8 & p \\
\hline PI & $1.11 \pm 0.32$ & $0.76 \pm 0.25$ & $<0.001$ & $0.68 \pm 0.21$ & $<0.001$ & $0.66 \pm 0.21$ & $<0.001$ \\
\hline GI & $1.42 \pm 0.33$ & $1.05 \pm 0.37$ & $<0.001$ & $1.00 \pm 0.36$ & $<0.001$ & $0.95 \pm 0.34$ & $<0.001$ \\
\hline BOP & $46.15 \pm 24.8$ & $25.33 \pm 12.3$ & $<0.001$ & $24.25 \pm 10.3$ & $<0.001$ & $23.30 \pm 10.6$ & $<0.001$ \\
\hline
\end{tabular}

The p-value was calculated by repeat analysis of variance (ANOVA) combined with Greenhouse-Geisser correction and Bonferroni correction. A significance level of $5 \%$ was used.

\subsection{Whole mouth PD and CAL}

Firstly, a whole mouth evaluation was brought out in order to consider an overall treating result of PVP-I. At T0, the mean PD was 3.04 \pm 0.73 . Significant improvements were noted at all 3 following appointments. In those 3 reevaluations, the PD was noted as $2.52 \pm 0.59$ at T4; $2.46 \pm 0.77$ at T6 and $2.43 \pm 0.84$ at T8 (Table 2). A reduction was observed in PD measures of $0.53 \pm 0.32$ at $\mathrm{T} 4$. The reduction was noted as $0.58 \pm 0.46$ at T6. And at T8, the mean reduction was $0.61 \pm 0.39$ (Table 3). For CAL, it changed from $3.85 \pm 1.00$ at T0 to $3.24 \pm 0.52$ at T8 (Table 2). Statistic presented a gain of $0.49 \pm 0.48$ at $\mathrm{T} 4,0.57 \pm 0.34$ at $\mathrm{T} 6$ and $0.61 \pm 0.33$ at $\mathrm{T} 8$ (Table 3). Significant differences in CAL at baseline and the 3 following time points were noted. 
Table 2. Clinical PD and CAL including whole mouth, moderately deep and deep pockets $($ mean \pm SD)

\begin{tabular}{|c|c|c|c|c|c|c|c|}
\hline & T0 & T4 & $\mathbf{p}$ & T6 & $\mathbf{p}$ & T8 & $\mathbf{p}$ \\
\hline \multicolumn{8}{|l|}{ Whole mouth } \\
\hline $\mathrm{PD}(\mathrm{mm})$ & $3.04 \pm 0.73$ & $2.52 \pm 0.59$ & $<0.001$ & $2.46 \pm 0.77$ & $<0.001$ & $2.43 \pm 0.84$ & $<0.001$ \\
\hline $\mathrm{CAL}(\mathrm{mm})$ & $3.85 \pm 1.00$ & $3.36 \pm 0.81$ & $<0.001$ & $3.28 \pm 0.51$ & $<0.001$ & $3.24 \pm 0.52$ & $<0.001$ \\
\hline \multicolumn{8}{|c|}{ Moderately deep pockets $(\mathrm{PD} \geq 5$ and $\mathrm{PD}<7)$} \\
\hline $\mathrm{PD}(\mathrm{mm})$ & $5.23 \pm 0.42$ & $3.73 \pm 0.87$ & $<0.001$ & $3.63 \pm 0.86$ & $<0.001$ & $3.62 \pm 0.87$ & $<0.001$ \\
\hline CAL (mm) & $5.78 \pm 1.22$ & $4.29 \pm 1.46$ & $<0.001$ & $4.24 \pm 1.46$ & $<0.001$ & $4.22 \pm 1.46$ & $<0.001$ \\
\hline \multicolumn{8}{|c|}{ Deep pockets $(\mathrm{PD} \geq 7)$} \\
\hline $\mathrm{PD}(\mathrm{mm})$ & $7.78 \pm 0.79$ & $6.02 \pm 1.09$ & $<0.001$ & $6.00 \pm 1.09$ & $<0.001$ & $5.99 \pm 1.07$ & $<0.001$ \\
\hline $\mathrm{CAL}(\mathrm{mm})$ & $8.64 \pm 1.36$ & $6.94 \pm 1.37$ & $<0.001$ & $6.90 \pm 1.37$ & $<0.001$ & $6.89 \pm 1.35$ & $<0.001$ \\
\hline
\end{tabular}

The p-value was calculated by repeat analysis of variance (ANOVA) combined with Greenhouse-Geisser correction and Bonferroni correction. A significance level of 5\% was used.

Table 3. Changes in PD and CAL from baseline to reevaluating appointments including whole mouth, moderately deep and deep pockets $($ mean \pm SD)

\begin{tabular}{|c|c|c|c|c|}
\hline & Change (T0 to T4) & Change (T0 to T6) & Change (T0 to T8) & $\mathbf{p}$ \\
\hline \multicolumn{5}{|c|}{ Whole mouth } \\
\hline PD (mm) & $0.53 \pm 0.32$ & $0.58 \pm 0.46$ & $0.61 \pm 0.39$ & $<0.001$ \\
\hline CAL (mm) & $0.49 \pm 0.48$ & $0.57 \pm 0.34$ & $0.61 \pm 0.33$ & $<0.001$ \\
\hline \multicolumn{5}{|c|}{ Moderately deep pockets $(P D \geq 5$ and $P D<7)$} \\
\hline PD (mm) & $1.50 \pm 0.81$ & $1.6 \pm 0.86$ & $1.61 \pm 0.85$ & $<0.001$ \\
\hline CAL (mm) & $1.50 \pm 0.79$ & $1.55 \pm 0.78$ & $1.57 \pm 0.79$ & $<0.001$ \\
\hline \multicolumn{5}{|c|}{ Deep pockets (PD $\geq 7)$} \\
\hline PD (mm) & $1.75 \pm 0.91$ & $1.78 \pm 0.90$ & $1.79 \pm 0.90$ & $<0.001$ \\
\hline CAL (mm) & $1.69 \pm 1.13$ & $1.73 \pm 1.12$ & $1.74 \pm 1.12$ & $<0.001$ \\
\hline
\end{tabular}

The p-value was calculated by repeat analysis of variance (ANOVA) combined with Greenhouse-Geisser correction and Bonferroni correction. A significance level of 5\% was used. 


\subsection{Analysis of moderately deep pockets $(\mathrm{PD} \geqslant 5$ and $\mathrm{PD}<7)$}

We should also consider the analysis of PD and CAL in moderately deep and deep pockets, which is one of the most important symptoms of chronic periodontitis. The comparison of $\mathrm{PD}$ and $\mathrm{CAL}$ at moderately deep pockets before and after treatment also revealed a significant difference. Mean PD and CAL at moderately deep pockets were noted as $5.23 \pm 0.42$ and $5.78 \pm 1.22$ at $\mathrm{T} 0$, which remarkably changed into $3.62 \pm 0.87$ and $4.22 \pm 1.46$ at $\mathrm{T} 8$ (Table 2). Statistic also presented a gain of $1.61 \pm 0.85$ in PD and $1.57 \pm 0.79$ in CAL at T8 (Table 3).

\subsection{Analysis of deep pockets $(P D \geqslant 7)$}

When analyzing deep pockets, statistic remarkability was noted. At T0, the mean $\mathrm{PD}$ was $7.78 \pm 0.79$. In those 3 reevaluations, the $\mathrm{PD}$ was noted as $6.02 \pm 1.09$ at $\mathrm{T} 4$, $6.00 \pm 1.09$ at T6 and $5.99 \pm 1.07$ at T8 (Table 2). A reduction was observed in PD measures of $1.75 \pm 0.91$ at T4. The reduction was noted as $1.78 \pm 0.90$ at T6. And at $\mathrm{T} 8$, the mean reduction was $1.79 \pm 0.90$ (Table 3 ).

For CAL, it changed from $8.64 \pm 1.36$ at T0 to $6.89 \pm 1.35$ at T8 (Table 2). Statistic presented a gain of $1.69 \pm 1.13$ at T4, $1.73 \pm 1.12$ at T6 and $1.74 \pm 1.12$ at T8 (Table 3). Significant differences in CAL at baseline and the 3 following time points were noted.

Additionally, when comparing the results from table 3, it should also be noted that the reduction in $\mathrm{PD}$ and $\mathrm{CAL}$ at deep pockets was more significant than shallow ones: $1.79 \pm 0.90$ and $1.74 \pm 1.12$ in deep pockets compare to $1.61 \pm 0.85$ and $1.57 \pm 0.79$ in moderately deep pockets and $0.61 \pm 0.39$ and $0.61 \pm 0.33$ in whole mouth. In other word, the deeper the pockets are, the more pronounced the effectiveness of PVP-I is.

\subsection{BANA Test}

At T8, the results came up with significant reduce in bacteria level from $1.67 \pm 0.48$ at baseline to $0.81 \pm 0.48$ at $\mathrm{T} 4$, to $0.63 \pm 0.49$ at $\mathrm{T} 6$ and to $0.39 \pm 0.49$ at $\mathrm{T} 8$ (Table 4).

Table 4. BANA test score (mean \pm SD)

\begin{tabular}{|c|c|c|c|c|c|c|c|}
\hline & T0 & T4 & p & T6 & p & T8 & p \\
\hline BANA score & $1.67 \pm 0.48$ & $0.81 \pm 0.48$ & $<0.001$ & $0.63 \pm 0.49$ & $<0.001$ & $0.39 \pm 0.49$ & $<0.001$ \\
\hline
\end{tabular}

The p-value was calculated by repeat analysis of variance (ANOVA) combined with Greenhouse-Geisser correction and Bonferroni correction. A significance level of 5\% was used.

\section{Discussion}

The findings from the present study demonstrated that the topical application of $0.1 \%$ PVP-I, used as subgingival irrigation along with subgingival root debridement improved the outcome of nonsurgical periodontal therapy. At all 3 reevaluation appointments, all of the indices have significant improvements, especially the reduction of periodontal pockets and the gain of clinical attachment.

The current findings are in agreement with data revealed by Christersson et al. (1988) who assessed the effect of one episode of ultrasonic scaling with $0.5 \%$ PVP- 
I or saline used as cooling solutions on a number of parameters characteristic of periodontal disease in 19 subjects [24]. One year after treatment, it was reported that the percentage of sites with deep pockets $(>5 \mathrm{~mm})$ in the iodine group was reduced from $43 \%$ to $5 \%$. On the other hand, the change in the control group was $37 \%$ to $5 \%$. In the iodine-group $80 \%$ of sites with initially $>7 \mathrm{~mm}$ pockets gained $>2 \mathrm{~mm}$ probing attachment. The corresponding figure in the saline group was $55 \%$. By that point, it was shown that $0.5 \%$ PVP-I has a remarkable effect in gaining clinical attachment.

Moreover, comparison between effects of 0.5\%PVP-I in non-surgical treatment and surgical methods was also noticed. It was reported by Forabosco et al. (1996) [25]. They compared in 8 subjects the outcomes of (i) surgical access flap (modified Widman flap) + conventional root planing and (ii) non-surgical root planing with an ultrasonic instrument connected to a container housing a $0.5 \%$ PVP-I solution. After 12 months, final examination was performed on the patients. The authors concluded "At that stage, clinical evaluation revealed that the Odontoson MA plus an ionized irrigant solution achieved a statistically comparable outcome to that of surgical treatment, even in the pockets initially up to $7 \mathrm{~mm}$ in depth". Therefore, it is shown that $0.5 \%$ PVP-I as subgingival irrigation could bring out the results tantamount to those of surgical methods.

Our study also reported that $0.1 \%$ PVP-I might be more effective in deep pockets compared to shallow ones. In some respect, that finding is in agreement with data previously presented by Rosling et al. (1986) [26] that was used higher PVP-I concentration. They divided 20 volunteers into 2 groups: 10 in a test and 10 in a control group, to determine the effect of $0.5 \%$ PVP-I used as an adjunct to either surgical or non-surgical therapy. A split-mouth design was also used. In each subject, 2 quadrants were treated by non-surgical with $0.5 \%$ PVP-I meanwhile treatment in the 2 remaining quadrants included flap elevation and debridement. A 12-month post-treatment examination showed that the adjuvant had no effect on overall gingivitis or probing pocket depth reductions, in either the surgery or the scaling groups. There was, however, at deep pockets a more marked gain of probing attachment in the nonsurgical plus $0.5 \%$ PVP-I group than in the other 3 groups. As a result, it showed a more pronounced effectiveness of $0.5 \%$ PVP-I in deeper pockets.

In addition, in previous clinical trials (Rosling et al. 198626, Christersson et al. 1988 [24], Grossi et al. 1997 [27], Forabosco et al. 1996 [25]), a 0.5\% concentration of PVP-I was used through an ultrasonic device during non-surgical therapy. In the present study, a lower concentration of the agent was delivered. The decision to employ a $0.1 \%$ PVP-I solution was based on findings by Caufield et al. (1987) [28]. They used a membrane transfer technique to demonstrate that $0.1 \%$ to $0.5 \%$ solutions of iodine had a bactericidal effect on putative periodontal pathogens such as A. actinomycetemcomitans, P.gingivals and P. intermedia within a 5 minute period of exposure [20].

The BANA results of this trial indicated a reduction in bacteria level in patients' plaque. This somehow agree with Collins et al.'s study. They showed that the adjunctive use of $10 \%$ PVP-I irrigation was able to reduce subgingival P. gingivalis to below detectable levels in 10 of 11 culture-positive patients [29]. The fact that PVP-I subgingival irrigation prior to tooth extraction can reduce the incidence of 
bacteremia by $30-50 \%$ also points to the potential of PVP-I as a periodontal pocket disinfectant [30]. Cigana et al. found that 15 patients of daily subgingival irrigation with $10 \%$ PVP-I caused significantly more clinical and histological decrease in gingival inflammation than saline irrigation [31]. A new study of Sarhmann et al. (2015) also stated that bacteremia after subgingival instrumentation with concomitant 10\% PCP-I rinsing is reduced. Significantly less bacteria and bacteremia were found in test group compared to the control and anaerobic bacteria were not found in the test group [19]. In our study, we used lower PVP-I concentration (0.1\% PVPI) but the results also showed a remarkable decline in periodontitis-induced bacteria such as P. gingivalis, T. denticola and T. forsythia by BANA test after 8 week periodontitis treatment.

Furthermore, during this trial, subjects had a full oral treatment including scaling and root planing pocket debridement along with $0.1 \%$ PVP-I irrigation. Thus, multifaceted approaches to controlling periodontal diseases are being developed along both mechanical and pharmaceutical lines [32]. Cheetham et al. (1988) showed that lipopolysaccharide presence on periodontally involved roots was removed by only 15 lights, overlapping hand instrument strokes per root surface [33]. Similar results were verified by Smart et al. (1990) following a conservative ultrasonic instrumentation regimen consisting of light pressure for a short period of time, ensuring overlapping instrumentation [34]. For that reason, the improvements of clinical parameters might be influenced by not only PVP-I but also treatment procedures conducted by clinicians.

Moreover, patients also had oral hygiene instructions at least twice during 8week study. Therefrom, it could be suggested that the improved outcome of treatment was explained not only by an improved removal/disinfection of the pocket microbiota during the subgingival therapy, which is the results of the treatment procedures, but also by the decline of periodontal pathogens from other intra-oral sites, which results from patients' better oral hygiene. With better oral hygiene, periodontal disease-induced bacteria in oral cavity might decrease, thence, translocation of microorganisms to recently treated periodontal sites from saliva, tonsils and tongue might decline. In other word, effective periodontal therapy consists of professionally administered antimicrobial intervention including mechanical debridement, and well-tolerated antiseptic agents exhibiting high activity against a variety of periodontal pathogens and delivered in ways that simultaneously affect pathogens residing in different oral ecological niches, followed by a maintenance program having a strong anti-infective emphasis [18].

Our study has certain limitations. We only survey in patients who had PVP-I as their periodontal irrigating liquid. We didn't have a control group to compare the effectiveness of PVP-I. Suggestion is that another trial should be conducted in which there is a control group of which the patients have a different irrigation either as placebo-treating or treating liquid. Another limitation is that in comparison with other trials, our 8-week trial can be considered quite short. In other studies, the final examination is much longer from the initial visit such as 3 months (Zanatta 2006 [18]) or 1 year (Rosling et al. 2001 [20]). Short examination might cause missing out of significant important points. For that reason, future investigation should be longer. 


\section{Conclusions}

In conclusion, $0.1 \%$ PVP-I, as subgingival irrigation, was proved to have beneficial influences on chronic periodontitis treatments, particularly in reducing periodontal pocket depth and increasing clinical attachment. However, future studies with longer follow-up periods and a control group are needed to fully delineate the utility of PVP-I in the treatment of destructive periodontal diseases.

\section{References}

1. The American Academy of Periodontology. American Academy of Periodontology Task Force report on the update to the 1999 classification of periodontal diseases and conditions. J Periodontol. 2015;86(7):835-838.

2. Shewale AH, GAttAni DR, Bhatia N, Mahajan R, Saravanan SP. Prevalence of Periodontal Disease in the General Population of India-A Systematic Review. Journal of clinical and diagnostic research: JCDR. 2016 Jun;10(6): ZE04-ZE09.

3. Listgarten MA. Structure of the microbial flora associated with periodontal health and disease in man: a light and electron microscopic study. J Periodontol. 1976 Jan;47(1):118.

4. Ximénez - Fyvie LA, Haffajee AD, Socransky SS. Comparison of the microbiota of supra - and subgingival plaque in health and periodontitis. J Clin Periodontol. 2000;27(9):648-57.

5. Tanner A, Maiden MF, Macuch PJ, Murray LL, Kent RL. Microbiota of health, gingivitis, and initial periodontitis. J Clin Periodontol. 1998;25(2):85-98.

6. Tanner A, Kent R, Maiden MF, Taubman MA. Clinical, microbiological and immunological profile of healthy, gingivitis and putative active periodontal subjects. J Periodont Res. 1996;31(3):195-204.

7. Ramberg P, Sekino S, Uzel NG, Socransky S, Lindhe J. Bacterial colonization during de novo plaque formation. Journal of clinical periodontology. 2003 Nov 1;30(11):990-5.

8. Socransky SS, Haffajee AD. Periodontal microbial ecology. Periodontoly 2000. 2005;38(1):135-87.

9. Holt SC, Ebersole JL. Porphyromonas gingivalis, Treponema denticola, and Tannerella forsythia: the 'red complex', a prototype polybacterial pathogenic consortium in periodontitis. Periodontology 2000. 2005;38(1):72-122.

10. Socransky SS, Smith C, Haffajee AD. Subgingival microbial profiles in refractory periodontal disease. J Clin Periodontol. 2002;29(3):260-8.

11. Sanz M, Lau L, Herrera D, Morillo JM, Silva A. Methods of detection of Actinobacillus actinomycetemcomitans, Porphyromonas gingivalis and Tannerella forsythensis in periodontal microbiology, with special emphasis on advanced molecular techniques: a review. J Clin Periodontol. 2004 Dec 1;31(12):1034-47.

12. Kaldahl WB, Kalkwarf KL, Patil KD, Molvar MP, Dyer JK. Long-term evaluation of periodontal therapy: I. Response to 4 therapeutic modalities. J Periodont. 1996;67(2):93102.

13. Ramfjord SP, Caffesse RG, Morrison EC, Hill RW, Kerry GJ, Appleberry EA, Nissle RR, Stults DL. 4 modalities of periodontal treatment compared over 5 years. J Clin Periodontol. $1987 ; 14(8): 445-52$.

14.Pihlstrom BL, McHuon RB, Oliphant TH, Ortiz - Campos C. Comparison of surgical and nonsurgical treatment of periodontal disease A review of current studies and additional results after 6 1/2 years. J Clin Periodontol. 1983;10(5):524-41.

15. Lindhe J, Nyman S, Karring T. Scaling and root planing in shallow pockets. J Clin Periodontol. 1982;9(5):415-8. 
16. Petersilka GJ, Ehmke B, Flemmig TF. Antimicrobial effects of mechanical debridement. Periodontology 2000. 2002 Jan 1;28(1):56-71.

17. Holt SC, Kesavalu L, Walker S, Genco CA. Virulence factors of Porphyromonas gingivalis. Periodontology 2000. 1999 Jun 1;20(1):168-238.

18.Zanatta GM, Bittencourt S, Nociti FH, Sallum EA, Sallum AW, Casati MZ. Periodontal Debridement with Povidone - Iodine in Periodontal Treatment: Short - Term Clinical and Biochemical Observations. J Periodontol. 2006;77(3):498-505.

19. Sahrmann P, Manz A, Attin T, Zbinden R, Schmidlin PR. Effect of application of a PVP - iodine solution before and during subgingival ultrasonic instrumentation on post treatment bacteraemia: a randomized single - centre placebo - controlled clinical trial. J Clin Periodont. 2015;42(7):632-9.

20. Rosling B, Hellström MK, Ramberg P, Socransky SS, Lindhe J. The use of PVP - iodine as an adjunct to non - surgical treatment of chronic periodontitis. J Clin Periodontol. 2001;28(11):1023-31.

21. Sahrmann P, Imfeld T, Ronay V, Attin T, Schmidlin PR. Povidone-iodine gel and solution as adjunct to ultrasonic debridement in nonsurgical periodontitis treatment: an RCT pilot study. Quintessence Int. 2014;45(4).

22. Reimer K, Schreier H, Erdos G, König B, König W, Fleischer W. Molecular effects of a microbicidal substance on relevant microorganisms: electron microscopic and biochemical studies on povidone-iodine. Int J Of Hyg Environment Med. 1998;200(5-6):423-34.

23. Loesche WJ, Lopatin DE, Giordano J, Alcoforado G, Hujoel P. Comparison of the benzoyl-DL-arginine-naphthylamide (BANA) test, DNA probes, and immunological reagents for ability to detect anaerobic periodontal infections due to Porphyromonas gingivalis, Treponema denticola, and Bacteroides forsythus. J Clin Microbiol. 1992 Feb 1;30(2):427-33.

24. Christersson LA, Rosling BG, Dunford RG, Wikesjö UM, Zambon JJ, Genco RJ. Monitoring of subgingival Bacteroides gingivalis and Actinobacillus actinomycetemcomitans in the management of advanced periodontitis. Adv Dental Res. 1988v;2(2):382-8.

25. Forabosco A, Galetti R, Spinato S, Colao P, Casolari C. A comparative study of a surgical method and scaling and root planing using the Odontoson ${ }^{\circledR}$. J Clin Periodontol. 1996;23(7):611-4.

26. Rosling BG, Slots J, Christersson LA, Gröndahl HG, Genco RJ. Topical antimicrobial therapy and diagnosis of subgingival bacteria in the management of inflammatory periodontal disease. J Clin Periodontol. 1986;13(10):975-81.

27. Grossi SG, Skrepcinski FB, DeCaro T, Robertson DC, Ho AW, Dunford RG, Genco RJ. Treatment of periodontal disease in diabetics reduces glycated hemoglobin. J Periodontol. 1997;68(8):713-9.

28. Caufield PW, Allen DN, Childers NK. In vitro susceptibilities of suspected periodontopathic anaerobes as determined by membrane transfer assay. Antimicro Agents Chemother. 1987;31(12):1989-93.

29. Collins JG, Offenbacher S, Arnold RR. Effects of a combination therapy to eliminate Porphyromonas gingivalis in refractory periodontitis. J Periodontol. 1993 Oct;64(10):998-1007.

30. Hoang T, Jorgensen MG, Keim RG, Pattison AM, Slots J. Povidone - iodine as a periodontal pocket disinfectant. J Periodont Res. 2003;38(3):311-7.

31. Cigana F, Kerebel B, David J, Doumenjou F, Da Costa Noble R. A clinical and histological study of the efficacy of betadine on gingival inflammation. $\mathrm{J}$ de biologie buccale. 1991;19(2):173-84.

32. Slots J. Primer for antimicrobial periodontal therapy. J Periodont Res. 2000 Apr $1 ; 35(2): 108-14$ 
33. Cheetham WA, Wilson M, Kieser JB. Root surface debridement-an in vitro assessment. J Clin Periodontol. 1988 May 1;15(5):288-92.

34. Smart GJ, Wilson M, Davies EH, Kieser JB. The assessment of ultrasonic root surface debridement by determination of residual endotoxin levels. J Clin Periodontol. 1990;17(3):174-8. 